\title{
Mixed Caputo Fractional Neutral Stochastic Differential Equations with Impulses and Variable Delay
}

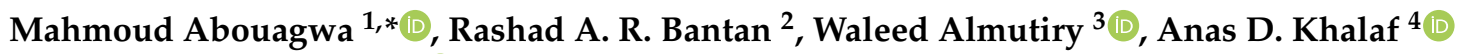 \\ and Mohammed Elgarhy ${ }^{5}$ \\ 1 Department of Mathematical Statistics, Faculty of Graduate Studies for Statistical Research, Cairo University, \\ Giza 12613, Egypt \\ 2 Department of Marine Geology, Faulty of Marine Science, King AbdulAziz University, \\ Jeddah 21551, Saudi Arabia; rbantan@kau.edu.sa \\ 3 Department of Mathematics, College of Science and Arts in Ar Rass, Qassim University, \\ Buryadah 52571, Saudi Arabia; wkmtierie@qu.edu.sa \\ 4 Minsitry of Education, General Directorate of Education in Saladin, Tikrit 34001, Iraq; \\ anasdheyab@hust.edu.cn \\ 5 The Higher Institute of Commercial Sciences, Al Mahalla Alkubra, Algarbia 31951, Egypt; \\ m_elgarhy85@sva.edu.eg \\ * Correspondence: mahmoud.aboagwa@cu.edu.eg
}

check for updates

Citation: Abouagwa, M.; Bantan, R.A.R.; Almutiry, W.; Khalaf, A.D.; Elgarhy, M. Mixed Caputo Fractional Neutral Stochastic Differential Equations with Impulses and Variable Delay. Fractal Fract. 2021, 5, 239. https://doi.org/10.3390/ fractalfract5040239

Academic Editors: Giacomo Ascione, Alessandra Meoli and Enrica Pirozzi

Received: 12 September 2021

Accepted: 11 November 2021

Published: 23 November 2021

Publisher's Note: MDPI stays neutral with regard to jurisdictional claims in published maps and institutional affiliations.

Copyright: (c) 2021 by the authors. Licensee MDPI, Basel, Switzerland. This article is an open access article distributed under the terms and conditions of the Creative Commons Attribution (CC BY) license (https:/ / creativecommons.org/licenses/by/ $4.0 /)$.

\begin{abstract}
In this manuscript, a new class of impulsive fractional Caputo neutral stochastic differential equations with variable delay (IFNSDEs, in short) perturbed by fractional Brownain motion (fBm) and Poisson jumps was studied. We utilized the Carathéodory approximation approach and stochastic calculus to present the existence and uniqueness theorem of the stochastic system under Carathéodory-type conditions with Lipschitz and non-Lipschitz conditions as special cases. Some existing results are generalized and enhanced. Finally, an application is offered to illustrate the obtained theoretical results.
\end{abstract}

Keywords: fractional differential system of neutral type; fractional brownian motion; fractional calculus; Poisson jump; Carathéodory approximation

\section{Introduction}

The theory of fractional differential equations is an important component in the fractional calculus and plays a key role in helping researchers to explore the hidden properties of the dynamics of complex systems in viscoelasticity, electromagnetism, diffusion, mechanics, control, signal processing, physics, and many other fields [1-5]. The most important advantage of utilizing systems of fractional order in the applications is their non-local property. Recently, random fluctuations have appeared commonly in various natural and synthetic systems, and fractional stochastic differential equations (FSDEs) with random perturbations have been applied as the mathematical models of many practical systems. This motivates researchers to move from fractional deterministic models to fractional stochastic models to guarantee the model performance. The theory of FSDEs is of interest because of the applications in many fields of engineering and science such as mechanics, control, physics, economics and many other areas [6-8]. A considerable amount of literature has been published on the existence and uniqueness of solutions for FSDEs. One can see [9-17] and the references therein.

On the other hand, in many mathematical models, the claims often display longrange memories, possibly due to extreme weather or natural disasters, and in some cases, many dynamical systems depend not only on present and past states but also contain the derivatives with delays. Neutral functional differential equations are often used to describe such systems [18]. In recent decades, the existence and uniqueness of mild solutions for fractional neutral SDEs have attracted much attention. For instance, Lakhel 
and Mckibben [19] established the existence of mild solutions for a class of FNSDEs driven by $\mathrm{fBm}$ with infinite delay; Dhanalakshmi and Balasubramaniam [20] showed the stability result of higher-order FNSDEs with infinite delay driven by Poisson jumps and the Rosenblatt process; Ramkumar et al. [21] obtained the existence and optimal control for a class of Caputo FNSDEs driven by fBm and Poisson jumps; Alnafisah and Ahmed [22] proved the existence and controllability for neutral delay Hilfer fractional integrodifferential equations driven with $\mathrm{fBm}$ by means of the fixed point theorem and semigroup theory.

The theory of impulsive differential equations is developing as an active area of investigation due to the applications in engineering, biology, physics, and many other areas [23-25]. The systems with impulses are utilized for studying the dynamics of processes subject to abrupt changes at discrete moments. Very recently, impulsive FSDEs (IFSDEs) arising in a very natural way as mathematical models are often applied to describe the case where deterministic changes with impulses are interwoven with noisy fluctuations. For more details on the existence and uniqueness for IFSDEs, see [26-30] and the references cited therein. Dhanalaksmi and Balasubramaniam [31] utilized the existence and exponential stability of mild solutions for impulsive fractional neutral SDEs driven by fBm in Hilbert space. Muthukumar and Thiagu [32] derived the existence and approximate controllability of solutions to fractional neutral impulsive SDEs of order $1<q \leqslant 2$ with infinite delay and Poisson jumps.

Based on the above and to the best of our knowledge, there is no manuscript considering the solvability of an impulsive Caputo fractional neutral stochastic system driven by both $\mathrm{fBm}$ and Poisson jumps. In order to fill this gap, we considered the following IFNSDEs with variable delay driven by fBm and Poisson jumps in Hilbert space:

$$
\left\{\begin{aligned}
\begin{array}{rl}
D_{t}^{\beta}[y(t)-f(t, y(t-\rho(t)))]= & A[y(t)-f(t, y(t-\rho(t)))]+\Gamma_{t}^{1-\beta}\left[g(s, y(s), y(s-\rho(s))) \frac{d w^{H}(t)}{d t}\right. \\
& \left.\left.\quad+\int_{Z} h(t, y(t), y(t-\rho(t)), \eta)\right) \tilde{N}(d t, d \eta)\right], t \in[0, b], t \neq t_{k}
\end{array} \\
\begin{array}{rl}
\triangle y\left(t_{k}\right)=y\left(t_{k}^{+}\right)-y\left(t_{k}^{-}\right)=I_{k}\left(y\left(t_{k}\right)\right), \quad t=t_{k} \quad k=1,2, \ldots, q, q \in \mathbb{N} \\
y(t)=\varrho(t), \quad-\tau \leqslant t \leqslant 0,
\end{array}
\end{aligned}\right.
$$

where $D_{t}^{\beta}$ is the Caputo fractional derivative of order $\beta, 0<\beta<1$. $\Gamma_{t}^{1-\beta}$ (.) denotes the $1-\beta$ order fractional integral. Let $A: \mathbb{D}(A) \subset \mathcal{X} \longrightarrow \mathcal{X}$ be an infinitesimal generator of a solution operator $\left\{S_{\beta}(t)\right\}_{t \geqslant 0}$ defined on a Hilbert space $\mathcal{X}$ endowed by the inner product $\langle.,$.$\rangle and norm \|.\|_{\mathcal{X}} . w^{H}$ is a $\mathrm{fBm}$ with Hurst parameter $1 / 2<H<1$ defined on a real separable Hilbert space $\mathcal{Y}$. Let $f:[0, b] \times \mathcal{X} \longrightarrow \mathcal{X}, g:[0, b] \times \mathcal{X} \times \mathcal{X} \longrightarrow \mathcal{L}_{2}^{0}(\mathcal{Y}, \mathcal{X})$ and $h:[0, b] \times \mathcal{X} \times \mathcal{X} \times Z \longrightarrow \mathcal{X}$ be nonlinear functions. Let $\wp=\wp([-\tau, 0] ; \mathcal{X})$ be the Banach space of all continuous functions $\varrho:[-\tau, 0] \longrightarrow \mathcal{X}$ endowed by the norm

$$
\|\varrho\|=\sup \{\|\varrho(\theta)\|:-\tau \leqslant \theta \leqslant 0\}
$$

and the initial data $y(0)=\varrho=\{\varrho(\theta):-\tau \leqslant \theta \leqslant 0\}$ is an $\mathcal{F}_{0}$-measurable $\wp([-\tau, 0], \mathcal{X})$ valued random variable such that $\mathbb{E}\|\varrho\|^{2}<\infty$. $\tilde{N}(d t, d \eta)=N(d t, d \eta)-\tilde{\lambda}(d \eta) d t$ is the Poisson counting measure. Let $\mathcal{M}_{2}([-\tau, b], \mathcal{X})$ be the space of all $\mathcal{X}$-valued $\mathcal{F}_{t}$-adapted processes $\{y(t),-\tau \leqslant t \leqslant b\}$ endowed with the norm $\mathbb{E}\|y\|_{\mathcal{M}_{2}}^{2}=\mathbb{E}\|\varphi\|^{2}+\mathbb{E} \int_{0}^{b}\|y(t)\|^{2} d t<$ $\infty$. Here, $I_{k}: \mathcal{X} \longrightarrow \mathcal{X}, k=1,2, \ldots, q$ are bounded functions with the fixed times $t_{k}$ satisfying $0=t_{0}<t_{1}<t_{2}<\ldots<t_{q}<b$, and $y\left(t_{k}^{+}\right)$and $y\left(t_{k}^{-}\right)$represent the right and left limits of $y(t)$ at time $t_{k}$, respectively. Further, $\Delta y\left(t_{j}\right)=y\left(t_{k}^{+}\right)-y\left(t_{k}^{-}\right)$determines the jump in the state $y$ at time $t_{k}$, where $I_{k}$ is the jump size.

It is noted that when $\rho(t)=I_{k}=0$, our model (1) reduces to the [21] model, which has been studied by means of successive approximation under non-Lipschitz conditions. In contrast, we used the Carathéodory approximation approach to obtain the existence of the unique mild solution for Equation (1) under Carathéodory conditions with the non-Lipschitz condition used in [21] as a special case. Therefore, some results in [21] are generalized and enhanced. We highlight the contributions of this article as follows. 
- This article model's IFNSDEs are more general than the [21] model as it takes the variable delays described by the term $\rho(t)$ and possible jumps shown as impulses into consideration.

- It is noted that the proofs of Theorem 3.3 in [21] and Theorem 3 in [33] are proved by means of successive approximation. However, in our case here, Theorem 3.1 is proved by means of the Carathéodory approximation approach, which is more complicated.

- Our Carathéodory conditions are more general than the non-Lipschitz condition used in [21] and contain it as special case. Hence, some results in [21] are generalized and extended.

This article is arranged as follows. In Section 2, we review some preliminary notions and notations about stochastic integral with respect to $\mathrm{fBm}$ as well as fractional calculus and state our assumptions. Section 3 is devoted to proving our main theorem of the existence and uniqueness of mild solutions to Equation (1). Then, an application to validate our research is discussed in Section 4. Finally, the conclusion is given in Section 5.

\section{Preliminaries}

Through the present section, we collect some notions and notations needed to establish our main result. Assume $(\Omega, \mathcal{F}, \mathbb{P})$ is a filtered probability space with $\mathcal{F}_{0}$ containing all $\mathbb{P}$-null sets. The $\mathrm{fBm}$ with $H \in\left(\frac{1}{2}, 1\right)$ is a centered process of Gaussian type $w^{H}=$ $\left\{w^{H}(t)\right\}_{0 \leqslant t \leqslant T}$ with the following variance-covariance function:

$$
K_{H}(u, v)=\mathbb{E}\left(w^{H}(u) w^{H}(v)\right)=\frac{1}{2}\left(u^{2 H}+v^{2 H}-|u-v|^{2 H}\right), u, v \in(-\infty, \infty)
$$

and second partial derivative [19]:

$$
\frac{\partial K_{H}}{\partial u \partial v}=(2 H-2) H|u-v|^{2 H-2}, \quad H>\frac{1}{2}
$$

So, we can write:

$$
K_{H}(u, v)=(2 H-2) H \int_{0}^{u} \int_{0}^{v}\left|u_{1}-v_{1}\right|^{2 H-2} d u_{1} d v_{1} .
$$

For any real and separable Hilbert spaces $\mathcal{X}$ and $\mathcal{Y}$, assume $\mathcal{L}(\mathcal{Y}, \mathcal{X})$ is the space of all bounded linear operators from $\mathcal{Y}$ to $\mathcal{X}$. Let $\mathbb{Q} \in \mathcal{L}(\mathcal{Y}, \mathcal{X})$ be the operator defined by $\mathbb{Q} e_{n}=\lambda_{n} e_{n}$ with finite trace $\operatorname{tr} \mathbb{Q}=\sum_{n=1}^{\infty} \lambda_{n}<\infty$, for $\lambda_{n} \geqslant 0(n=1,2, \ldots)$, which are non-negative real numbers and $\left\{e_{n}\right\}$, which is a complete orthonormal basis in $\mathcal{Y}$. The infinite dimensional $\mathrm{fBm}$ on $\mathcal{Y}$ is defined as

$$
w^{H}(t)=w_{\mathbb{Q}}^{H}(t)=\sum_{n=1}^{\infty} \sqrt{\lambda_{n}} \cdot e_{n} \cdot w_{n}^{H}(t)
$$

with real independent fBm's $w_{n}^{H}$. Construct the space $\mathcal{L}_{2}^{0}:=\mathcal{L}_{2}^{0}(\mathcal{Y}, \mathcal{X})$ of all HilbertSchmidt operators $\zeta: \mathcal{Y} \longrightarrow \mathcal{X}$, equipped with the inner product $\langle\varphi, \zeta\rangle_{\mathcal{L}_{2}^{0}}=\sum_{n=1}^{\infty}\left\langle\varphi e_{n}, \zeta e_{n}\right\rangle$ and norm:

$$
\|\zeta\|_{\mathcal{L}_{2}^{0}}^{2}:=\sum_{n=1}^{\infty}\left\|\sqrt{\lambda_{n}} \zeta e_{n}\right\|^{2}<\infty
$$

For any $\phi(s) \in \mathcal{L}_{2}^{0}(\mathcal{Y}, \mathcal{X}), s \in[0, T]$ such that $\sum_{n=1}^{\infty}\left\|R^{*} \phi \mathbb{Q}^{\frac{1}{2}} e_{n}\right\|_{\mathcal{L}_{2}^{0}}^{2}<\infty$, the Weiner integral of $\phi$ with respect to $w^{H}$ is defined by:

$$
\int_{0}^{t} \phi(s) d w^{H}(s)=\sum_{n=1}^{\infty} \int_{0}^{t} \sqrt{\lambda_{n}} \phi(s) e_{n} d w_{n}^{H}(s) .
$$


Lemma 1 ([20]). For any $\phi:[0, b] \longrightarrow \mathcal{L}_{2}^{0}(\mathcal{Y}, \mathcal{X})$ with $\int_{0}^{b}\|\phi(s)\|_{\mathcal{L}_{2}^{0}}^{2} d s<\infty$, satisfying Equation (2), what follows is satisfied:

$$
\mathbb{E}\left\|\int_{0}^{t} \phi(s) d w^{H}(s)\right\|^{2} \leqslant 2 H t^{2 H-1} \int_{0}^{t}\|\phi(s)\|_{\mathcal{L}_{2}^{0}}^{2} d s .
$$

We refer to [34-38] for more details on the stochastic integral with respect to fBm.

Definition $1([3,5])$. The $\beta$-order fractional integral of Riemann-Lioville sense for $g:[0, b] \longrightarrow \mathcal{X}$ is expressed by:

$$
J_{t}^{\beta} g(t)=\frac{1}{\Gamma(\beta)} \int_{0}^{t}(t-s)^{\beta-1} g(s) d s, \quad \beta>0 .
$$

Definition 2 ([39]). The Caputo $\beta$-order derivative with a 0 lower bound for $g:[0, b] \longrightarrow \mathcal{X}$ is expressed as:

$$
D_{t}^{\beta} g(t)=\frac{1}{\Gamma(k-\beta)} \int_{0}^{t} \frac{g^{(k)}(s)}{(t-s)^{\beta+1-k}} d s=J_{t}^{k-\beta} g^{(k)}(t), \quad 0<k-1<\beta<k, t \geqslant 0 .
$$

For further discussion on the fractional Riemann-Liouville and Caputo derivatives, one can refer to $[3,5,39]$.

Next, a two-parameter Mittag-Leffler function is defined by the series expansion:

$$
E_{\beta, \alpha}(w)=\sum_{k=0}^{\infty} \frac{w^{k}}{\Gamma(\beta k+\alpha)}=\frac{1}{2 \pi i} \int_{c} \frac{\lambda^{\beta-\alpha} e^{\lambda}}{\lambda^{\beta}-w} d \lambda, \quad \alpha, \beta>0, w \in \mathbb{C}
$$

where $\mathbb{C}$ is a contour that starts and ends with $-\infty$ and encircles the disk $|\lambda| \leqslant|w|^{\frac{1}{2}}$ counterclockwise.

Following Definition 2.9 of [21], we constructed the definition of the mild solution for Equation (1) as:

Definition 3. The mild solution $y:[-\tau, b] \longrightarrow \mathcal{X}$ of (1) is a stochastic process satisfying:

(i) $y(t)$ is Cadlag and $\mathcal{F}_{t}$-adapted.

(ii) $\int_{0}^{b} \mathbb{E}\|y(s)\|^{2} d s<\infty$, a.s.

(iii) The coming integral equation is true.

$$
\begin{aligned}
& y(t)=\varrho(t), \quad t \in[-\tau, 0], \\
& y(t)= S_{\beta}(t)[\varrho(0)-f(0, \varrho)]+f(t, y(t-\rho(t))) \\
&++\int_{0}^{t} S_{\beta}(t-s) g(s, y(s), y(s-\rho(s))) d w^{H}(s) \\
&+\int_{0}^{t} \int_{Z} S_{\beta}(t-s) h(s, y(s), y(s-\rho(s)), \eta) \tilde{N}(d s, d \eta) \\
&+\sum_{0<t_{k}<t} S_{\beta}\left(t-t_{k}\right) I_{k}\left(y\left(t_{k}\right)\right), \quad t \in[0, b],
\end{aligned}
$$

where $S_{\beta}(t)$ is the solution operator generated by $A$ and given by:

$$
S_{\beta}(t)=E_{\beta, 1}\left(A t^{\beta}\right)=\frac{1}{2 \pi i} \int_{\hat{B}_{r}} e^{\mu t} \frac{\mu^{\beta-1}}{\mu^{\beta}-A} d \mu .
$$

(iv) $P\{y(t)=z(t), \forall 0 \leqslant t \leqslant b\}=1$ if $z(t)$ is another solution to (1).

The coming assumptions on the coefficients of (1) were prepared for studying the existence and uniqueness of the mild solution. 
(A1). The infinitesimal generator $A: \mathbb{D} \subset \mathcal{X} \longrightarrow \mathcal{X}$ of a strong and continuous semigroup of bounded and linear operator $S_{\beta}(t)$, satisfying $S_{\beta}(0)=\mathbb{I}$ (the identity operator on $\mathcal{X}$ ); there exists some constant $M>0$ obeying:

$$
\left\|S_{\beta}(t)\right\| \leqslant M, \text { for all } t \in[0, b],
$$

(A2). There exists a function $\mathcal{K}(t, v):[0, b] \times[0, \infty) \longrightarrow[0, \infty)$, such that:

(a) $\mathcal{K}(t, v)$ is local and integrable in $t$ for all fixed $v \in[0, \infty)$ and is continuous, monotone and nondecreasing in $v$ for all fixed $t \in[0, b]$.

(b) Furthermore, for all fixed $t \in[0, b]$ and $x, y \in \wp$, this inequality is true:

$$
\begin{aligned}
\mathbb{E} \int_{0}^{t} & \|g(t, x, y)\|^{2} d s \vee \mathbb{E} \int_{0}^{t} \int_{Z}\|h(t, x, y, \eta)\|^{2} \hat{\lambda}(\eta) d s \\
& \vee \mathbb{E}\left(\int_{0}^{t} \int_{Z}\|h(t, x, y, \eta)\|^{4} \hat{\lambda}(\eta) d s\right)^{\frac{1}{2}} \leqslant \int_{0}^{t} \mathcal{K}\left(s, \mathbb{E}\|x\|^{2}+\mathbb{E}\|y\|^{2}\right) d s
\end{aligned}
$$

(c) For any positive constant $\gamma$, the deterministic equation

$$
\frac{d \vartheta}{d t}=\gamma \mathcal{K}(t, \vartheta), \quad t \in[0, b]
$$

has a global solution for some initial value $\vartheta_{0} \geqslant 0$.

(A3). There exists a function $\mathcal{M}(t, v):[0, b] \times[0, \infty) \longrightarrow[0, \infty)$, such that:

(a) $\mathcal{M}(t, v)$ is local and integrable in $t$ for any fixed $v \in[0, \infty)$ and is continuous, monotone, nondecreasing, and concave in $v$ for any fixed $t \geqslant 0$ such that $\mathcal{M}(t, 0)=0$ and $\int_{0+} \frac{1}{\mathcal{M}(t, v)} d v=+\infty$.

(b) Furthermore, for any fixed $t \in[0, b]$ and $x_{1}, x_{2}, y_{1}, y_{2} \in \wp$, this inequality holds:

$$
\begin{aligned}
\mathbb{E} \int_{0}^{t}\left\|g\left(t, x_{1}, x_{2}\right)-g\left(t, y_{1}, y_{2}\right)\right\|^{2} d s \\
\vee \mathbb{E} \int_{0}^{t} \int_{Z}\left\|h\left(t, x_{1}, x_{2}, \eta\right)-h\left(t, y_{1}, y_{2}, \eta\right)\right\|^{2} \hat{\lambda}(\eta) d s \\
\vee \mathbb{E}\left(\int_{0}^{t} \int_{Z}\left\|h\left(t, x_{1}, x_{2}, \eta\right)-h\left(t, y_{1}, y_{2}, \eta\right)\right\|^{4} \hat{\lambda}(\eta) d s\right)^{\frac{1}{2}} \\
\quad \leqslant \int_{0}^{t} \mathcal{M}\left(s, \mathbb{E}\left\|x_{1}-x_{2}\right\|^{2}+\mathbb{E}\left\|y_{1}-y_{2}\right\|^{2}\right) d s
\end{aligned}
$$

(c) If a non-negative continuous function $Y(t), t \in[0, b]$ satisfies

$$
\left\{\begin{array}{l}
Y(t) \leqslant \mathcal{N} \int_{0}^{t} \mathcal{M}(s, Y(s)) d s, \quad 0 \leqslant t \leqslant b \\
Y(0)=0
\end{array}\right.
$$

we have $Y(0) \equiv 0$ for all positive constant $\mathcal{N}$ and $0 \leqslant t \leqslant b$.

(A4). For some positive constant $C_{f}$ and $y_{1}, y_{2} \in \wp$,

$$
\left\|f\left(t, y_{1}\right)-f\left(t, y_{2}\right)\right\|^{2} \leqslant C_{f}\left\|y_{1}-y_{2}\right\|^{2} \text { and } f(t, 0)=0,
$$

for all $t \geqslant 0$.

(A5). There exists a constants $c_{k}>0$ such that for every $k=1,2, \ldots, q$,

$$
\left\|I_{k}\left(y_{1}\right)-I_{k}\left(y_{2}\right)\right\|^{2} \leqslant c_{k}\left\|y_{1}-y_{2}\right\|^{2} \text { and }\left\|I_{k}(0)\right\|^{2}=0,
$$


for all $y_{1}, y_{2} \in \wp$.

Remark 1. Let $\mathcal{M}(t, v)=B(t) \overline{\mathcal{M}}(v), t \in[0, b]$, where $B(t) \geqslant 0$ is locally integrable and $\overline{\mathcal{M}}(v)$ is a concave nondecreasing function from $[0, \infty[$ to $[0, \infty[$ such that $\overline{\mathcal{M}}(0)=0, \overline{\mathcal{M}}(v)>0$ for $v>0$ and $\int_{0^{+}} \frac{1}{\mathcal{M}(v)} d v=\infty$. Then, by the comparison theorem of differential equations, we know that condition $(A 3-c)$ holds.

Now, let us give some concrete examples of the function $\overline{\mathcal{M}}($.$) . Let \epsilon>0$ and let $\kappa \in(0,1)$ be sufficiently small. Define

$$
\begin{gathered}
\overline{\mathcal{M}}_{1}(v)=\epsilon v, \quad v \geqslant 0, \\
\overline{\mathcal{M}}_{2}(v)= \begin{cases}v \log \left(v^{-1}\right), & 0 \leqslant v \leqslant \kappa, \\
\kappa \log \left(\kappa^{-1}\right)+\overline{\mathcal{M}}_{2}(\kappa-)(v-\kappa), & v>\kappa,\end{cases}
\end{gathered}
$$

where $\overline{\mathcal{M}}_{2}$ denotes the derivative of function $\overline{\mathcal{M}}_{2}$. They are all concave nondecreasing functions satisfying $\int_{0^{+}} \frac{1}{\mathcal{M}_{i}(v)} d v=\infty(i=1,2)$. In particular, we see that the non-Lipschitz condition in [21] is a special case of our proposed condition.

\section{Existence and Uniqueness}

With the help of assumptions (A1)-(A5), we, through this section, develop the existence of the unique mild solution concerning Equation (1). Assume the Carathéodory approximation $y_{n}(t)$ is defined for all $b>0$ and any integer $n \geqslant 2 / \tau$ as follows:

$$
\begin{aligned}
& y_{n}(t)=\varrho(t), \quad-\tau \leqslant t \leqslant 0, \\
& y_{n}(t)= S_{\beta}(t)[\varrho(0)-f(0, \varrho)]+f\left(t, y_{n}(t-\rho(t))\right) \\
&+\int_{0}^{t} 1_{D_{n}^{c}}(s) S_{\beta}(t-s) g\left(s, y_{n}\left(s-\frac{1}{n}\right), y_{n}(s-\rho(s))\right) d w^{H}(s) \\
&+\int_{0}^{t} 1_{D_{n}^{c}}(s) S_{\beta}(t-s) h\left(s, y_{n}\left(s-\frac{1}{n}\right), y_{n}(s-\rho(s)), \eta\right) \tilde{N}(d s, d \eta) \\
&+\int_{0}^{t} 1_{D_{n}}(s) S_{\beta}(t-s) g\left(s, y_{n}\left(s-\frac{1}{n}\right), y_{n}\left(s-\rho(s)-\frac{1}{n}\right)\right) d w^{H}(s) \\
&+\int_{0}^{t} 1_{D_{n}}(s) S_{\beta}(t-s) h\left(s, y_{n}\left(s-\frac{1}{n}\right), y_{n}\left(s-\rho(s)-\frac{1}{n}\right), \eta\right) \tilde{N}(d s, d \eta) \\
&+\sum_{0<t_{k}<t} S_{\beta}\left(t-t_{k}\right) I_{k}\left(y_{n}\left(t_{k}-\frac{1}{n}\right)\right), \quad t \in[0, b],
\end{aligned}
$$

with the indicator functions $1_{D_{n}}$ and $1_{D_{n}^{c}}$ of $D_{n}=\left\{t: \rho(t)<\frac{1}{n}, 0 \leqslant t \leqslant b\right\}$ and $D_{n}^{c}=[0, b]-D_{n}$, respectively.

Theorem 1. Assume assumptions (A1)-(A5) are fulfilled. Suppose $\varrho$ is independent of the Poisson counting measure $\tilde{N}(d t, d \eta)$ and $f B m w^{H}(t)$. Then, provided $7 C_{f}+7 q M^{2} \sum_{k=1}^{q} c_{k}<1$, Equation (1) has a unique mild solution $y(t)$ on $\mathcal{M}_{2}([-\tau, b], \mathcal{X})$.

Proof. For the convenience of the readers, the proof is divided into the following steps. Step 1. This sequence $\left\{y_{n}(t)\right\}_{n \geqslant 2 / \tau}$ is claimed to be bounded.

By elementary inequality to (4), it is seen 


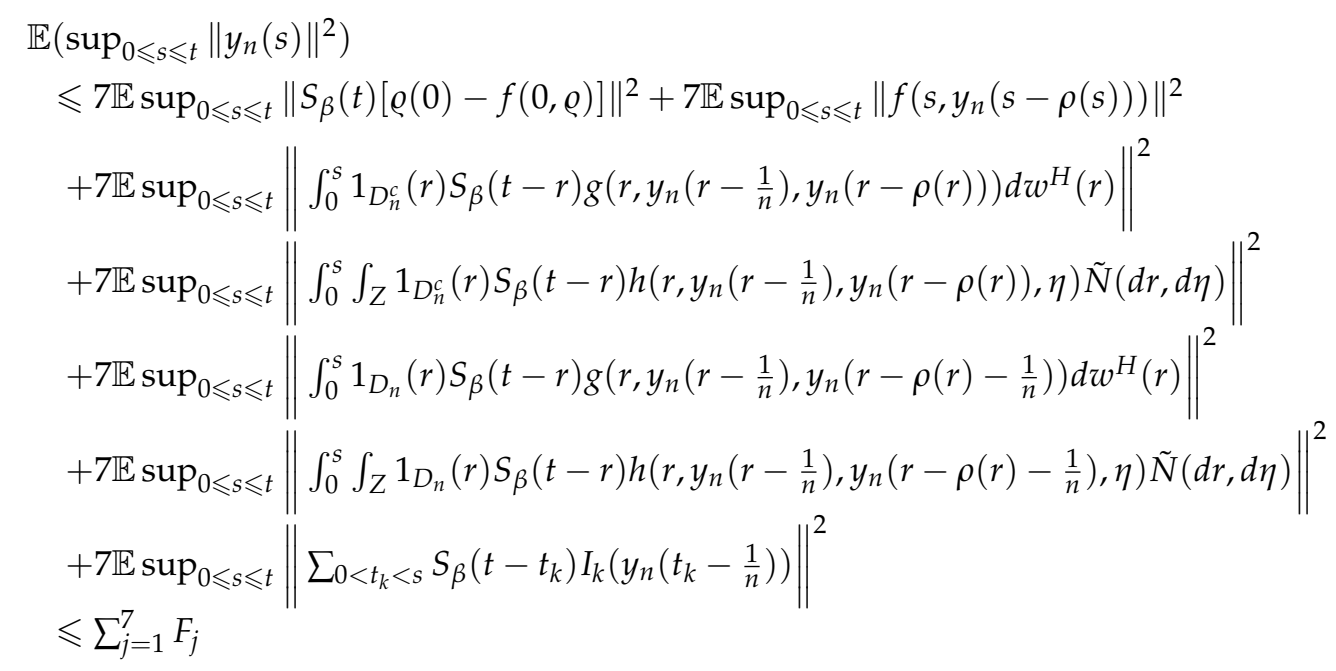

Thus, we have by conditions (A1) and (A4)

$$
F_{1} \leqslant 14 M^{2}\left[\mathbb{E}\|\varrho\|^{2}+C_{f} \mathbb{E}\|\varrho\|^{2}\right] \leqslant 14 M^{2}\left(1+C_{f}\right) \mathbb{E}\|\varrho\|^{2},
$$

and

$$
F_{2} \leqslant 7 \mathbb{E}\left\|f\left(t, y_{n}(t-\rho(t))\right)\right\|^{2} \leqslant 7 C_{f} \mathbb{E} \sup _{0 \leqslant s \leqslant t}\left\|y_{n}(s)\right\|^{2} .
$$

By Lemma 1 , Burkholder's inequality for pure jump stochastic integrals in $\mathcal{X}$ [40] and condition (A2), we obtain

$$
\begin{aligned}
F_{3}+F_{4} \leqslant & 14 M^{2} H t^{2 H-1} \mathbb{E} \int_{0}^{t} 1_{D_{n}^{c}}(s)\left\|g\left(s, y_{n}\left(s-\frac{1}{n}\right), y_{n}(s-\rho(s))\right)\right\|^{2} d s \\
& +7 M^{2} C_{b}\left[\mathbb{E} \int_{0}^{t} \int_{Z} 1_{D_{n}^{c}}(s)\left\|h\left(s, y_{n}\left(s-\frac{1}{n}\right), y_{n}(s-\rho(s)), \eta\right)\right\|^{2} \tilde{\lambda}(d \eta) d s\right. \\
& \left.+\mathbb{E}\left(\int_{0}^{t} \int_{Z} 1_{D_{n}^{c}}(s)\left\|h\left(s, y_{n}\left(s-\frac{1}{n}\right), y_{n}(s-\rho(s)), \eta\right)\right\|^{4} \hat{\lambda}(d \eta) d s\right)^{\frac{1}{2}}\right] \\
\leqslant & 7 M^{2}\left(C_{b}+2 H t^{2 H-1}\right) \int_{0}^{t} 1_{D_{n}^{c}}(s) \mathcal{K}\left(s, \mathbb{E}\left\|y_{n}\left(s-\frac{1}{n}\right)\right\|^{2}+\mathbb{E}\left\|y_{n}(s-\rho(s))\right\|^{2}\right) d s .
\end{aligned}
$$

Similarly,

$$
\begin{aligned}
& F_{5}+F_{6} \\
& \leqslant 7 M^{2}\left(C_{b}+2 H t^{2 H-1}\right) \int_{0}^{t} 1_{D_{n}}(s) \mathcal{K}\left(s, \mathbb{E}\left\|y_{n}\left(s-\frac{1}{n}\right)\right\|^{2}+\mathbb{E}\left\|y_{n}\left(s-\rho(s)-\frac{1}{n}\right)\right\|^{2}\right) d s .
\end{aligned}
$$

For $F_{7}$, we have by conditions (A1) and (A5)

$$
\begin{aligned}
F_{7} & \leqslant 7 q M^{2} \sum_{k=1}^{q} c_{k} \mathbb{E}\left\|y_{n}\left(t_{k}-\frac{1}{n}\right)\right\|^{2} \\
& \leqslant\left(7 q M^{2} \sum_{k=1}^{q} c_{k}\right) \mathbb{E} \sup _{0 \leqslant s \leqslant t}\left\|y_{n}(s)\right\|^{2} .
\end{aligned}
$$

Combining Equations (5)-(10), it concludes

$$
\begin{aligned}
& \mathbb{E}\left(\sup _{0 \leqslant s \leqslant t}\left\|y_{n}(s)\right\|^{2}\right) \\
& \leqslant \frac{14 M^{2}\left(1+C_{f}\right) \mathbb{E}\|\varrho\|^{2}}{1-7 C_{f}-7 q M^{2} \sum_{k=1}^{q} c_{k}}+\frac{7 M^{2}\left(C_{b}+2 H t^{2 H-1}\right)}{1-7 C_{f}-7 q M^{2} \sum_{k=1}^{q} c_{k}}
\end{aligned}
$$




$$
\begin{aligned}
& \times \int_{0}^{t} 1_{D_{n}^{c}}(s) \mathcal{K}\left(s, 2 \mathbb{E}\|\varrho\|^{2}+2 \mathbb{E} \sup _{0 \leqslant r \leqslant s}\left\|y_{n}(r)\right\|^{2}\right) d s \\
& +\frac{7 M^{2}\left(C_{b}+2 H t^{2 H-1}\right)}{1-7 C_{f}-7 q M^{2} \sum_{k=1}^{q} c_{k}} \int_{0}^{t} 1_{D_{n}}(s) \mathcal{K}\left(s, 2 \mathbb{E}\|\varrho\|^{2}+2 \mathbb{E} \sup _{0 \leqslant r \leqslant s}\left\|y_{n}(r)\right\|^{2}\right) d s \\
& \leqslant \frac{14 M^{2}\left(1+C_{f}\right) \mathbb{E}\|\rho\|^{2}}{1-7 C_{f}-7 q M^{2} \sum_{k=1}^{q} c_{k}}+\frac{7 M^{2}\left(C_{b}+2 H t^{2 H-1}\right)}{1-7 C_{f}-7 q M^{2} \sum_{k=1}^{q} c_{k}} \\
& \times \int_{0}^{t} \mathcal{K}\left(s, 2 \mathbb{E}\|\varrho\|^{2}+2 \mathbb{E} \sup _{0 \leqslant r \leqslant s}\left\|y_{n}(r)\right\|^{2}\right) d s .
\end{aligned}
$$

Hence, we have

$$
\begin{aligned}
2 \mathbb{E}\|\varrho\|^{2}+2 \mathbb{E}\left(\sup _{0 \leqslant s \leqslant t}\left\|y_{n}(s)\right\|^{2}\right) & \\
\leqslant & \frac{2\left[1+14 M^{2}\left(1+C_{f}\right)-7 C_{f}-7 q M^{2} \sum_{k=1}^{q} c_{k}\right]}{1-7 C_{f}-7 q M^{2} \sum_{k=1}^{q} c_{k}} \mathbb{E}\|\varrho\|^{2} \\
& +\frac{14 M^{2}\left(C_{b}+2 H t^{2 H-1}\right)}{1-7 C_{f}-7 q M^{2} \sum_{k=1}^{q} c_{k}} \int_{0}^{t} \mathcal{K}\left(s, 2 \mathbb{E}\|\varrho\|^{2}+2 \mathbb{E} \sup _{0 \leqslant r \leqslant s}\left\|y_{n}(r)\right\|^{2}\right) d s .
\end{aligned}
$$

Then, for any solution $\vartheta_{t}$, condition (A2-c) gives

$$
\begin{aligned}
\vartheta_{t} \leqslant & \frac{2\left[1+14 M^{2}\left(1+C_{f}\right)-7 C_{f}-7 q M^{2} \sum_{k=1}^{q} c_{k}\right]}{1-7 C_{f}-7 q M^{2} \sum_{k=1}^{q} c_{k}} \mathbb{E}\|\varrho\|^{2} \\
& +\frac{14 M^{2}\left(C_{b}+2 H t^{2 H-1}\right)}{1-7 C_{f}-7 q M^{2} \sum_{k=1}^{q} c_{k}} \int_{0}^{t} \mathcal{K}\left(s, \vartheta_{s}\right) d s .
\end{aligned}
$$

Since $\mathbb{E}\|\varrho\|^{2} \leqslant \infty$, it reads

$$
\mathbb{E}\left(\sup _{0 \leqslant s \leqslant t}\left\|y_{n}(s)\right\|^{2}\right) \leqslant \vartheta_{t} \leqslant \vartheta_{T}<\infty, \quad n \geqslant \frac{2}{n} .
$$

Therefore, $\left\{y_{n}(t)\right\}_{n \geqslant 2 / \tau}$ is uniformly bounded, and Step 1 is then fulfilled.

Step 2. For $s, t \in[0, b], s<t$ and $n \geqslant 2 / \tau$, it reads

$$
\mathbb{E}\left\|y_{n}(t)-y_{n}(s)\right\|^{2} \leqslant C_{1}\left\|S_{\beta}(t-s)-\mathbb{I}\right\|^{2}+C_{2}(t-s)+C_{3} \sum_{s<t_{k}<t} c_{k},
$$

where $C_{1}, C_{2}$, and $C_{3}$ are defined through the proof.

Note that:

$$
\begin{aligned}
& \mathbb{E}\left\|y_{n}(t)-y_{n}(s)\right\|^{2} \\
& \quad \leqslant 2 \mathbb{E}\left\|f\left(t, y_{n}(t-\rho(t))\right)-f\left(s, y_{n}(s-\rho(s))\right)\right\|^{2}+2 \mathbb{E}\left\|J_{n}(t, s)\right\|^{2} \\
& \quad \leqslant 2 C_{f} \mathbb{E}\left\|y_{n}(t)-y_{n}(s)\right\|^{2}+2 \mathbb{E}\left\|J_{n}(t, s)\right\|^{2} \\
& \quad \leqslant \frac{2}{1-2 C_{f}} \mathbb{E}\left\|J_{n}(t, s)\right\|^{2},
\end{aligned}
$$

where:

$$
\begin{aligned}
& \mathbb{E}\left\|J_{n}(t, s)\right\|^{2} \\
& \leqslant 5 \mathbb{E} \| \int_{0}^{s} 1_{D_{n}^{c}}(v)\left[S_{\beta}(t-v)-S_{\beta}(s-v)\right] g\left(v, y_{n}\left(v-\frac{1}{n}\right), y_{n}(v-\rho(u))\right) d w^{H}(v)
\end{aligned}
$$




$$
\begin{aligned}
& +\int_{s}^{t} 1_{D_{n}^{c}}(v) S_{\beta}(t-v) g\left(v, y_{n}\left(v-\frac{1}{n}\right), y_{n}(v-\rho(v))\right) d w^{H}(v) \|^{2} \\
& +5 \mathbb{E} \| \int_{0}^{s} \int_{Z} 1_{D_{n}^{c}}(v)\left[S_{\beta}(t-v)-S_{\beta}(s-v)\right] h\left(v, y_{n}\left(v-\frac{1}{n}\right), y_{n}(v-\rho(v)), \eta\right) \tilde{N}(d v, d \eta) \\
& +\int_{s}^{t} \int_{Z} 1_{D_{n}^{c}}(v) S_{\beta}(t-v) h\left(v, y_{n}\left(v-\frac{1}{n}\right), y_{n}(v-\rho(v)), \eta\right) \tilde{N}(d v, d \eta) \|^{2} \\
& +5 \mathbb{E} \| \int_{0}^{s} 1_{D_{n}}(v)\left[S_{\beta}(t-v)-S_{\beta}(s-v)\right] g\left(v, y_{n}\left(v-\frac{1}{n}\right), y_{n}\left(v-\rho(v)-\frac{1}{n}\right)\right) d w^{H}(v) \\
& +\int_{s}^{t} 1_{D_{n}}(v) S_{\beta}(t-v) g\left(v, y_{n}\left(v-\frac{1}{n}\right), y_{n}\left(v-\rho(v)-\frac{1}{n}\right)\right) d w^{H}(v) \|^{2} \\
& +5 \mathbb{E} \| \int_{0}^{s} \int_{Z} 1_{D_{n}}(v) \\
& \times\left[S_{\beta}(t-v)-S_{\beta}(s-v)\right] h\left(v, y_{n}\left(v-\frac{1}{n}\right), y_{n}\left(v-\rho(v)-\frac{1}{n}\right), \eta\right) \tilde{N}(d v, d \eta) \\
& +\int_{s}^{t} \int_{Z} 1_{D_{n}}(v) S_{\beta}(t-v) h\left(v, y_{n}\left(v-\frac{1}{n}\right), y_{n}\left(v-\rho(v)-\frac{1}{n}\right), \eta\right) \tilde{N}(d v, d \eta) \|^{2} \\
& +5 \mathbb{E} \| \sum_{0<t_{k}<s}\left[S_{\beta}\left(t-t_{k}\right)-S_{\beta}\left(s-t_{k}\right)\right] I_{k}\left(y_{n}\left(t_{k}-\frac{1}{n}\right)\right) \\
& +\sum_{s<t_{k}<t} S_{\beta}\left(t-t_{k}\right) I_{k}\left(y_{n}\left(t_{k}-\frac{1}{n}\right)\right) \|^{2}:=\sum_{i=1}^{5} J_{i} .
\end{aligned}
$$

Now for $J_{1}$, we have by Lemma 1 and conditions (A1) and (A2):

$$
\begin{aligned}
J_{1} \leqslant & 10 \mathbb{E}\left\|\int_{0}^{s} 1_{D_{n}^{c}}(v)\left[S_{\beta}(t-v)-S_{\beta}(s-v)\right] g\left(v, y_{n}\left(v-\frac{1}{n}\right), y_{n}(v-\rho(v))\right) d w^{H}(v)\right\|^{2} \\
& +10 \mathbb{E}\left\|\int_{s}^{t} 1_{D_{n}^{c}}(v) S_{\beta}(t-v) g\left(v, y_{n}\left(v-\frac{1}{n}\right), y_{n}(v-\rho(v))\right) d w^{H}(v)\right\|^{2} \\
\leqslant & 20 H s^{2 H-1}\left\|S_{\beta}(t-v)-S_{\beta}(s-v)\right\|^{2} \int_{0}^{s} 1_{D_{n}^{c}}(v) \\
& \times \mathcal{K}\left(v, \mathbb{E}\left\|y_{n}\left(v-\frac{1}{n}\right)\right\|^{2}+\mathbb{E}\left\|y_{n}(v-\rho(v))\right\|^{2}\right) d u \\
& +20 H(t-s)^{2 H-1}\left\|S_{\beta}(t-v)\right\|^{2} \int_{s}^{t} 1_{D_{n}^{c}}(v) \\
& \times \mathcal{K}\left(v, \mathbb{E}\left\|y_{n}\left(v-\frac{1}{n}\right)\right\|^{2}+\mathbb{E}\left\|y_{n}(v-\rho(v))\right\|^{2}\right) d v \\
\leqslant & 20 H s^{2 H-1} M^{2}\left\|S_{\beta}(t-s)-\mathbb{I}\right\|^{2} \int_{0}^{s} 1_{D_{n}^{c}}(v) \mathcal{K}\left(v, 2 \mathbb{E} \sup _{0 \leqslant r \leqslant v}\left\|y_{n}(r)\right\|^{2}\right) d v \\
& +20 H(t-s)^{2 H-1} M^{2} \int_{s}^{t} 1_{D_{n}^{c}}(v) \mathcal{K}\left(v, 2 \mathbb{E} \sup _{0 \leqslant r \leqslant v}\left\|y_{n}(r)\right\|^{2}\right) d v
\end{aligned}
$$

For $J_{2}$, we have by Burkholder's inequality and conditions (A1) and (A2):

$$
\begin{aligned}
J_{2} \leqslant & 10 \mathbb{E} \| \int_{0}^{s} \int_{Z} 1_{D_{n}^{c}}(v) \\
& \times\left[S_{\beta}(t-v)-S_{\beta}(s-v)\right] h\left(v, y_{n}\left(v-\frac{1}{n}\right), y_{n}(v-\rho(v)), \eta\right) \tilde{N}(d v, d \eta) \|^{2} \\
& +10 \mathbb{E}\left\|\int_{s}^{t} \int_{Z} 1_{D_{n}^{c}}(v) S_{\beta}(t-v) h\left(v, y_{n}\left(v-\frac{1}{n}\right), y_{n}(v-\rho(v)), \eta\right) \tilde{N}(d v, d \eta)\right\|^{2}
\end{aligned}
$$


$\leqslant 10 C_{b} M^{2}\left\|S_{\beta}(t-s)-\mathbb{I}\right\|^{2} \mathbb{E}\left[\int_{0}^{s} \int_{Z} 1_{D_{n}^{c}}(v)\left\|h\left(v, y_{n}\left(v-\frac{1}{n}\right), y_{n}(v-\rho(v)), \eta\right)\right\|^{2} \tilde{\lambda}(d \eta) d v\right.$

$\left.+\left(\int_{0}^{s} \int_{Z} 1_{D_{n}^{c}}(v)\left\|h\left(v, y_{n}\left(v-\frac{1}{n}\right), y_{n}(v-\rho(v)), \eta\right)\right\|^{4} \tilde{\lambda}(d \eta) d v\right)^{\frac{1}{2}}\right]$

$+10 C_{b} M^{2} \mathbb{E}\left[\int_{s}^{t} \int_{Z} 1_{D_{n}^{c}}(v)\left\|h\left(v, y_{n}\left(v-\frac{1}{n}\right), y_{n}(v-\rho(v)), \eta\right)\right\|^{2} \tilde{\lambda}(d \eta) d v\right.$

$\left.+\left(\int_{s}^{t} \int_{Z} 1_{D_{n}^{c}}(v)\left\|h\left(v, y_{n}\left(v-\frac{1}{n}\right), y_{n}(v-\rho(v)), \eta\right)\right\|^{4} \tilde{\lambda}(d \eta) d v\right)^{\frac{1}{2}}\right]$

$\leqslant 10 C_{b} M^{2}\left\|S_{\beta}(t-s)-\mathbb{I}\right\|^{2} \int_{0}^{s} 1_{D_{n}^{c}}(v) \mathcal{K}\left(v, 2 \mathbb{E} \sup _{0 \leqslant r \leqslant v}\left\|y_{n}(r)\right\|^{2}\right) d v$

$+10 C_{b} M^{2} \int_{s}^{t} 1_{D_{n}^{c}}(v) \mathcal{K}\left(v, 2 \mathbb{E} \sup _{0 \leqslant r \leqslant v}\left\|y_{n}(r)\right\|^{2}\right) d v$

Similarly, for $J_{3}$ and $J_{4}$, we have:

$$
\begin{aligned}
J_{3}+J_{4} \leqslant & 10 M^{2}\left\|S_{\beta}(t-s)-\mathbb{I}\right\|^{2}\left[C_{b}+2 H s^{2 H-1}\right] \int_{0}^{s} 1_{D_{n}}(v) \mathcal{K}\left(v, 2 \mathbb{E} \sup _{0 \leqslant r \leqslant v}\left\|y_{n}(r)\right\|^{2}\right) d v \\
& +10 M^{2}\left[C_{b}+2 H(t-s)^{2 H-1}\right] \int_{s}^{t} 1_{D_{n}}(v) \mathcal{K}\left(v, 2 \mathbb{E} \sup _{0 \leqslant r \leqslant v}\left\|y_{n}(r)\right\|^{2}\right) d v .
\end{aligned}
$$

For $J_{5}$, we have by conditions (A1) and (A5) and Hölder's inequality:

$$
\begin{aligned}
J_{5} \leqslant & 10 \mathbb{E}\left\|\sum_{0<t_{k}<s}\left(S_{\beta}\left(t-t_{k}\right)-S_{\beta}\left(s-t_{k}\right)\right) I_{k}\left(y_{n}\left(t_{k}-\frac{1}{n}\right)\right)\right\|^{2} \\
& +10 \mathbb{E}\left\|\sum_{s<t_{k}<t} S_{\beta}\left(t-t_{k}\right) I_{k}\left(y_{n}\left(t_{k}-\frac{1}{n}\right)\right)\right\|^{2} \\
\leqslant & 10 q M^{2}\left\|S_{\beta}(t-s)-\mathbb{I}\right\|^{2} \sum_{k=1}^{q} c_{k} \mathbb{E}\left\|y_{n}\left(t_{k}-\frac{1}{n}\right)\right\|^{2} \\
& +10 q M^{2} \sum_{s<t_{k}<t} c_{k} \mathbb{E}\left\|y_{n}\left(t_{k}-\frac{1}{n}\right)\right\|^{2} \\
\leqslant & 10 q M^{2}\left\|S_{\beta}(t-s)-\mathbb{I}\right\|^{2} \sum_{k=1}^{q} c_{k} \mathbb{E} \sup _{0 \leqslant s \leqslant t}\left\|y_{n}(s)\right\|^{2} \\
& +10 q M^{2} \sum_{s<t_{k}<t} c_{k} \mathbb{E} \sup _{0 \leqslant s \leqslant t}\left\|y_{n}(s)\right\|^{2} .
\end{aligned}
$$

Combining Equations (12)-(16) and using Step 1, we have:

$$
\begin{aligned}
\mathbb{E}\left\|J_{n}(t, s)\right\|^{2} \leqslant & 10 M^{2}\left\|S_{\beta}(t-s)-\mathbb{I}\right\|^{2}\left[C_{b}+2 H s^{2 H-1}\right] \int_{0}^{s} 1_{D_{n}^{c}}(v) \mathcal{K}\left(v, 2 \mathbb{E} \sup _{0 \leqslant r \leqslant v}\left\|y_{n}(r)\right\|^{2}\right) d v \\
& +10 M^{2}\left[C_{b}+2 H(t-s)^{2 H-1}\right] \int_{s}^{t} 1_{D_{n}^{c}}(v) \mathcal{K}\left(v, 2 \mathbb{E} \sup _{0 \leqslant r \leqslant v}\left\|y_{n}(r)\right\|^{2}\right) d v \\
& +10 M^{2}\left\|S_{\beta}(t-s)-\mathbb{I}\right\|^{2}\left[C_{b}+2 H s^{2 H-1}\right] \int_{0}^{s} 1_{D_{n}}(v) \mathcal{K}\left(v, 2 \mathbb{E} \sup _{0 \leqslant r \leqslant v}\left\|y_{n}(r)\right\|^{2}\right) d v \\
& +10 M^{2}\left[C_{b}+2 H(t-s)^{2 H-1}\right] \int_{s}^{t} 1_{D_{n}}(v) \mathcal{K}\left(v, 2 \mathbb{E} \sup _{0 \leqslant r \leqslant v}\left\|y_{n}(r)\right\|^{2}\right) d v \\
& +10 q M^{2}\left\|S_{\beta}(t-s)-\mathbb{I}\right\|^{2} \sum_{k=1}^{q} c_{k} \mathbb{E} \sup _{0 \leqslant s \leqslant t}\left\|y_{n}(s)\right\|^{2}
\end{aligned}
$$




$$
\begin{aligned}
& +10 q M^{2} \sum_{s<t_{k}<t} c_{k} \mathbb{E} \sup _{0 \leqslant s \leqslant t}\left\|y_{n}(s)\right\|^{2} \\
\leqslant & 10 M^{2}\left\|S_{\beta}(t-s)-\mathbb{I}\right\|^{2}\left[C_{b}+2 H s^{2 H-1}\right] \int_{0}^{s} \mathcal{K}\left(v, 2 \mathbb{E} \sup _{0 \leqslant r \leqslant v}\left\|y_{n}(r)\right\|^{2}\right) d v \\
& +10 M^{2}\left[C_{b}+2 H(t-s)^{2 H-1}\right] \int_{s}^{t} \mathcal{K}\left(v, 2 \mathbb{E} \sup _{0 \leqslant r \leqslant v}\left\|y_{n}(r)\right\|^{2}\right) d v \\
& +10 q M^{2}\left\|S_{\beta}(t-s)-\mathbb{I}\right\|^{2} \sum_{k=1}^{q} c_{k} \mathbb{E} \sup _{0 \leqslant s \leqslant t}\left\|y_{n}(s)\right\|^{2} \\
& +10 q M^{2} \sum_{s<t_{k}<t} c_{k} \mathbb{E} \sup _{0 \leqslant s \leqslant t}\left\|y_{n}(s)\right\|^{2} \\
\leqslant & 10 M^{2}\left[s\left(C_{b}+2 H s^{2 H-1}\right) \sup _{0 \leqslant v \leqslant s} \mathcal{K}(v, 2 C)+q C \sum_{k=1}^{q} c_{k}\right]\left\|S_{\beta}(t-s)-\mathbb{I}\right\|^{2} \\
& +10 M^{2}\left[C_{b}+2 H(t-s)^{2 H-1}\right] \sup _{0 \leqslant v \leqslant t} \mathcal{K}(v, 2 C)(t-s)+10 q C M^{2} \sum_{s<t_{k}<t} c_{k},
\end{aligned}
$$

where the constant $C$ comes from Step 1. Inserting Equation (17) in Equation (11), the required result is obtained with constants:

$$
\begin{gathered}
C_{1}=\frac{20 M^{2}\left[s\left(C_{b}+2 H s^{2 H-1}\right) \sup _{0 \leqslant v \leqslant s} \mathcal{K}(v, 2 C)+q C \sum_{k=1}^{q} c_{k}\right]}{1-2 M_{f}}, \\
C_{2}=\frac{20 M^{2}\left[C_{b}+2 H(t-s)^{2 H-1}\right] \sup _{0 \leqslant v \leqslant t} \mathcal{K}(v, 2 C)}{1-2 M_{f}}, \text { and } C_{3}=\frac{20 q C M^{2}}{1-2 C_{f}}
\end{gathered}
$$

Step 3. It is proved that $\left\{y_{n}(t)\right\}_{n \geqslant 2 / \tau}$ is Cauchy in $\mathcal{M}_{2}([-\tau, b], \mathbb{X})$. Using Equation (4), for $m>n \geqslant 2 / \tau$, it is easy to obtain:

$$
\mathbb{E}\left(\sup _{0 \leqslant s \leqslant t}\left\|y_{m}(s)-y_{n}(s)\right\|^{2}\right) \leqslant \frac{2}{1-2 C_{f}} \mathbb{E}\left(\sup _{0 \leqslant s \leqslant t}\left\|\mathrm{Y}_{n}(s)\right\|^{2}\right),
$$

where:

$$
\begin{aligned}
Y_{n}(t)= & \int_{0}^{t}\left\{1_{D_{m}^{c}}(s) S_{\beta}(t-s) g\left(s, y_{m}\left(s-\frac{1}{m}\right), y_{m}(s-\rho(s))\right)\right. \\
& \left.-1_{D_{n}^{c}}(s) S_{\beta}(t-s) g\left(s, y_{n}\left(s-\frac{1}{n}\right), y_{n}(s-\rho(s))\right)\right\} d w^{H}(s) \\
& +\int_{0}^{t} \int_{Z}\left\{1_{D_{m}^{c}}(s) S_{\beta}(t-s) h\left(s, y_{m}\left(s-\frac{1}{m}\right), y_{m}(s-\rho(s)), \eta\right)\right. \\
& \left.-1_{D_{n}^{c}}(s) S_{\beta}(t-s) h\left(s, y_{n}\left(s-\frac{1}{n}\right), y_{n}(s-\rho(s)), \eta\right)\right\} \tilde{N}(d s, d \eta) \\
& +\int_{0}^{t}\left\{1_{D_{m}}(s) S_{\beta}(t-s) g\left(s, y_{m}\left(s-\frac{1}{m}\right), y_{m}\left(s-\rho(s)-\frac{1}{m}\right)\right)\right. \\
& \left.-1_{D_{n}}(s) S_{\beta}(t-s) g\left(s, y_{n}\left(s-\frac{1}{n}\right), y_{n}\left(s-\rho(s)-\frac{1}{n}\right)\right)\right\} d w^{H}(s) \\
& +\int_{0}^{t} \int_{Z}\left\{1_{D_{m}}(s) S_{\beta}(t-s) h\left(s, y_{m}\left(s-\frac{1}{m}\right), y_{m}\left(s-\rho(s)-\frac{1}{m}\right), \eta\right)\right. \\
& \left.-1_{D_{n}}(s) S_{\beta}(t-s) h\left(s, y_{n}\left(s-\frac{1}{n}\right), y_{n}\left(s-\rho(s)-\frac{1}{n}\right), \eta\right)\right\} \tilde{N}(d s, d \eta)
\end{aligned}
$$




$$
+\sum_{0<t_{k}<t} S_{\beta}(t-s)\left[I_{k}\left(y_{m}\left(t_{k}-\frac{1}{m}\right)\right)-I_{k}\left(y_{n}\left(t_{k}-\frac{1}{n}\right)\right)\right]=\sum_{i=1}^{5} \Theta_{i} .
$$

The integrals above are computed by the technique of plus and minus:

$$
\begin{aligned}
& \Theta_{1}=\int_{0}^{t} 1_{D_{m}^{c}}(s) S_{\beta}(t-s) g\left(s, y_{m}\left(s-\frac{1}{m}\right), y_{m}(s-\rho(s))\right) d w^{H}(s) \\
& -\int_{0}^{t} 1_{D_{n}^{c}}(s) S_{\beta}(t-s) g\left(s, y_{n}\left(s-\frac{1}{n}\right), y_{n}(s-\rho(s))\right) d w^{H}(s) \\
& +\int_{0}^{t} 1_{D_{n}^{c}}(s) S_{\beta}(t-s) g\left(s, y_{m}\left(s-\frac{1}{m}\right), y_{m}(s-\rho(s))\right) d w^{H}(s) \\
& -\int_{0}^{t} 1_{D_{n}^{c}}(s) S_{\beta}(t-s) g\left(s, y_{m}\left(s-\frac{1}{m}\right), y_{m}(s-\rho(s))\right) d w^{H}(s) \\
& +\int_{0}^{t} 1_{D_{n}^{c}}(s) S_{\beta}(t-s) g\left(s, y_{n}\left(s-\frac{1}{m}\right), y_{n}(s-\rho(s))\right) d w^{H}(s) \\
& -\int_{0}^{t} 1_{D_{n}^{c}}(s) S_{\beta}(t-s) g\left(s, y_{n}\left(s-\frac{1}{m}\right), y_{n}(s-\rho(s))\right) d w^{H}(s) \\
& =\int_{0}^{t} 1_{D_{n}^{c}}(s) S_{\beta}(t-s)\left\{g\left(s, y_{m}\left(s-\frac{1}{m}\right), y_{m}(s-\rho(s))\right)\right. \\
& \left.-g\left(s, y_{n}\left(s-\frac{1}{m}\right), y_{n}(s-\rho(s))\right)\right\} d w^{H}(s) \\
& +\int_{0}^{t} 1_{D_{n}^{c}}(s) S_{\beta}(t-s)\left\{g\left(s, y_{n}\left(s-\frac{1}{m}\right), y_{n}(s-\rho(s))\right)\right. \\
& \left.-g\left(s, y_{n}\left(s-\frac{1}{n}\right), y_{n}(s-\rho(s))\right)\right\} d w^{H}(s) \\
& +\int_{0}^{t} 1_{D_{m}^{c}-D_{n}^{c}}(s) S_{\beta}(t-s) g\left(s, y_{m}\left(s-\frac{1}{m}\right), y_{m}(s-\rho(s))\right) d w^{H}(s) .
\end{aligned}
$$

Taking expectation, elementary inequality, Lemma 1, conditions (A1)-(A3), and Step 1, we have:

$$
\begin{aligned}
\mathbb{E} \sup _{0 \leqslant s \leqslant t}\left\|\Theta_{1}\right\|^{2} \leqslant & 6 H t^{2 H-1} M^{2} \int_{0}^{t} 1_{D_{n}^{c}}(s) \mathcal{M}\left(s, \mathbb{E}\left\|y_{m}\left(s-\frac{1}{m}\right)-y_{n}\left(s-\frac{1}{m}\right)\right\|^{2}\right. \\
& \left.+\mathbb{E}\left\|y_{m}(s-\rho(s))-y_{n}(s-\rho(s))\right\|^{2}\right) d s \\
& +6 H t^{2 H-1} M^{2} \int_{0}^{t} 1_{D_{n}^{c}}(s) \mathcal{M}\left(s, \mathbb{E}\left\|y_{n}\left(s-\frac{1}{m}\right)-y_{n}\left(s-\frac{1}{n}\right)\right\|^{2}\right) d s \\
& +6 H t^{2 H-1} M^{2} \int_{0}^{t} 1_{D_{m}^{c}-D_{n}^{c}}(s) \mathcal{K}\left(s, \mathbb{E}\left\|y_{m}\left(s-\frac{1}{m}\right)\right\|^{2}\right. \\
& \left.+\mathbb{E}\left\|y_{m}(s-\rho(s))\right\|^{2}\right) d s \\
\leqslant & 6 H t^{2 H-1} M^{2} \int_{0}^{t} 1_{D_{n}^{c}}(s) \mathcal{M}\left(s, 2 \mathbb{E} \sup _{0 \leqslant v \leqslant s}\left\|y_{m}(v)-y_{n}(v)\right\|^{2}\right) d s \\
& +6 H t^{2 H-1} M^{2} \int_{0}^{b} 1_{D_{n}^{c}}(s) \mathcal{M}\left(s, \mathbb{E}\left\|y_{n}\left(s-\frac{1}{m}\right)-y_{n}\left(s-\frac{1}{n}\right)\right\|^{2}\right) d s \\
& +6 H t^{2 H} M^{2}\left(\sup _{0 \leqslant s \leqslant t} \mathcal{K}(s, 2 C)\right) \mu\left(D_{m}^{c}-D_{n}^{c}\right) .
\end{aligned}
$$

Similarly, we have for $\Theta_{2}$ by Burkholder's inequality, conditions (A1)-(A3) and Step 1: 


$$
\begin{aligned}
\mathbb{E} \sup _{0 \leqslant s \leqslant t}\left\|\Theta_{2}\right\|^{2} \leqslant & 3 \mathbb{E} \sup _{0<s<t} \| \int_{0}^{s} \int_{Z} 1_{D_{n}^{c}}(v) S_{\beta}(s-v)\left\{h\left(v, y_{m}\left(v-\frac{1}{m}\right), y_{m}(v-\rho(v)), \eta\right)\right. \\
& \left.-h\left(v, y_{n}\left(v-\frac{1}{m}\right), y_{n}(v-\rho(v)), \eta\right)\right\} \tilde{N}(d v, d \eta) \|^{2} \\
& +3 \mathbb{E} \sup _{0<s<t} \| \int_{0}^{s} \int_{Z} 1_{D_{n}^{c}}(v) S_{\beta}(s-v)\left\{h\left(v, y_{n}\left(v-\frac{1}{m}\right), y_{n}(v-\rho(v)), \eta\right)\right. \\
& \left.-h\left(v, y_{n}\left(v-\frac{1}{n}\right), y_{n}(v-\rho(v)), \eta\right)\right\} \tilde{N}(d v, d \eta) \|^{2} \\
& +3 \mathbb{E} \sup _{0<s<t} \| \int_{0}^{s} \int_{Z} 1_{D_{m}^{c}-D_{n}^{c}(v) S_{\beta}(s-v)} \\
& \times h\left(v, y_{m}\left(v-\frac{1}{m}\right), y_{m}(v-\rho(v)), \eta\right) \tilde{N}(d v, d \eta) \|^{2} \\
\leqslant & 3 C_{b} M^{2} \int_{0}^{t} 1_{D_{n}^{c}}(s) \mathcal{M}\left(s, 2 \mathbb{E} \sup _{0 \leqslant v \leqslant s}\left\|y_{m}(v)-y_{n}(v)\right\|^{2}\right) d s \\
& +3 C_{b} M^{2} \int_{0}^{b} 1_{D_{n}^{c}}(s) \mathcal{M}\left(s, \mathbb{E}\left\|y_{n}\left(s-\frac{1}{m}\right)-y_{n}\left(s-\frac{1}{n}\right)\right\|^{2}\right) d s \\
& +3 C_{b} M^{2}\left(\sup _{0 \leqslant s \leqslant b} \mathcal{K}(s, 2 C) \mu\left(D_{m}^{c}-D_{n}^{c}\right) .\right.
\end{aligned}
$$

By similar analysis for $\Theta_{3}$ and $\Theta_{4}$, we obtain:

$$
\begin{aligned}
\mathbb{E} \sup _{0 \leqslant s \leqslant t}\left(\left\|\Theta_{3}\right\|^{2}+\left\|\Theta_{4}\right\|^{2}\right) \\
\leqslant 3 M^{2}\left(C_{b}+2 H t^{2 H-1}\right) \int_{0}^{t} 1_{D_{m}}(s) \mathcal{M}\left(s, 2 \mathbb{E} \sup _{0 \leqslant v \leqslant s}\left\|y_{m}(v)-y_{n}(v)\right\|^{2}\right) d s \\
\quad+3 M^{2}\left(C_{b}+2 H t^{2 H-1}\right) \int_{0}^{b} 1_{D_{m}}(s) \mathcal{M}\left(s, \mathbb{E}\left\|y_{n}\left(s-\frac{1}{m}\right)-y_{n}\left(s-\frac{1}{n}\right)\right\|^{2}\right. \\
\left.\quad+\mathbb{E}\left\|y_{n}\left(s-\rho(s)-\frac{1}{m}\right)-y_{n}\left(s-\rho(s)-\frac{1}{n}\right)\right\|^{2}\right) d s \\
\quad+3 M^{2}\left(C_{b}+2 H t^{2 H}\right)\left(\sup _{0 \leqslant s \leqslant b} \mathcal{K}(s, 2 C)\right) \mu\left(D_{n}-D_{m}\right) .
\end{aligned}
$$

For $\Theta_{5}$, we have by the technique of plus and minus, conditions (A1) and (A5):

$$
\begin{aligned}
\underset{0 \leqslant s \leqslant t}{\mathbb{E} \sup _{0 \leqslant s}\left\|\Theta_{5}\right\|^{2} \leqslant} & 2 q M^{2} \sum_{k=1}^{q} c_{k} \mathbb{E}\left\|y_{m}\left(t_{k}-\frac{1}{m}\right)-y_{n}\left(t_{k}-\frac{1}{m}\right)\right\|^{2} \\
& +2 q M^{2} \sum_{k=1}^{q} c_{k} \mathbb{E}\left\|y_{n}\left(t_{k}-\frac{1}{m}\right)-y_{n}\left(t_{k}-\frac{1}{n}\right)\right\|^{2} \\
\leqslant & 2 q M^{2} \sum_{k=1}^{q} c_{k} \mathbb{E} \sup _{0 \leqslant s \leqslant t}\left\|y_{m}(s)-y_{n}(s)\right\|^{2} \\
& +2 q M^{2} \sum_{k=1}^{q} c_{k} \mathbb{E}\left\|y_{n}\left(t_{k}-\frac{1}{m}\right)-y_{n}\left(t_{k}-\frac{1}{n}\right)\right\|^{2}
\end{aligned}
$$

Collecting (18)-(23), we find:

$\mathbb{E}\left(\sup _{0 \leqslant s \leqslant t}\left|y_{m}(s)-y_{n}(s)\right|^{2}\right) \leqslant C_{4} \int_{0}^{t} \mathcal{M}\left(s, 2 \mathbb{E} \sup _{0 \leqslant v \leqslant s}\left\|y_{m}(v)-y_{n}(v)\right\|^{2}\right) d s$ 


$$
\begin{aligned}
& +C_{4} \int_{0}^{b} 1_{D_{n}^{c}}(s) \mathcal{M}\left(s, \mathbb{E}\left\|y_{n}\left(s-\frac{1}{m}\right)-y_{n}\left(s-\frac{1}{n}\right)\right\|^{2}\right) d s \\
& +C_{4} \int_{0}^{b} 1_{D_{m}}(s) \mathcal{M}\left(s, \mathbb{E}\left\|y_{n}\left(s-\frac{1}{m}\right)-y_{n}\left(s-\frac{1}{n}\right)\right\|^{2}\right. \\
& \left.+\mathbb{E}\left\|y_{n}\left(s-\rho(s)-\frac{1}{m}\right)-y_{n}\left(s-\rho(s)-\frac{1}{n}\right)\right\|^{2}\right) d s \\
& +C_{6} \mathbb{E}\left\|y_{n}\left(t_{k}-\frac{1}{m}\right)-y_{n}\left(t_{k}-\frac{1}{n}\right)\right\|^{2} \\
& +C_{5}\left(\sup _{0 \leqslant s \leqslant b} \mathcal{K}(s, 2 C)\right) \mu\left(D_{n}-D_{m}\right)
\end{aligned}
$$

where $C_{4}=\frac{30 M^{2}\left(C_{b}+2 H t^{2 H-1}\right)}{1-2 C_{f}-24 q M^{2} \sum_{k=1}^{q} c_{k}}, C_{5}=\frac{60 M^{2}\left(C_{b}+2 H t^{2 H}\right)}{1-2 C_{f}-24 q M^{2} \sum_{k=1}^{q} c_{k}}$ and $C_{6}=\frac{24 q M^{2} \sum_{k=1}^{q} c_{k}}{1-2 C_{f}-24 q M^{2} \sum_{k=1}^{q} c_{k}}$.

In the lines that follow, we can estimate:

$$
\begin{aligned}
\int_{0}^{b} 1_{D_{n}^{c}}(s) \mathcal{M}\left(s, \mathbb{E}\left\|y_{n}\left(s-\frac{1}{m}\right)-y_{n}\left(s-\frac{1}{n}\right)\right\|^{2}\right) d s \\
=\int_{0}^{\frac{1}{n}} 1_{D_{n}^{c}}(s) \mathcal{M}\left(s, \mathbb{E}\left\|y_{n}\left(s-\frac{1}{m}\right)-y_{n}\left(s-\frac{1}{n}\right)\right\|^{2}\right) d s \\
\quad+\int_{\frac{1}{n}}^{b} 1_{D_{n}^{c}}(s) \mathcal{M}\left(s, \mathbb{E}\left\|y_{n}\left(s-\frac{1}{m}\right)-y_{n}\left(s-\frac{1}{n}\right)\right\|^{2}\right) d s \\
\leqslant \int_{0}^{\frac{1}{n}} 1_{D_{n}^{c}}(s) \mathcal{M}\left(s, 2 \mathbb{E}\left\|y_{n}\left(s-\frac{1}{m}\right)\right\|^{2}+2 \mathbb{E}\left\|y_{n}\left(s-\frac{1}{n}\right)\right\|^{2}\right) d s \\
\quad+b \mathcal{M}\left(s, C_{1}\left\|S_{\beta}\left(\frac{1}{n}-\frac{1}{m}\right)-\mathbb{I}\right\|^{2}+C_{2}\left(\frac{1}{n}-\frac{1}{m}\right)+C_{3} \sum_{s-\frac{1}{n}<t_{k}<s-\frac{1}{m}} c_{k}\right) \\
\leqslant \mathcal{M}\left(s, 4 \mathbb{E}\|\varrho\|^{2}+4 C\right) \frac{1}{n} \\
\quad+b \mathcal{M}\left(s, C_{1}\left\|S_{\beta}\left(\frac{1}{n}-\frac{1}{m}\right)-\mathbb{I}\right\|^{2}+C_{2}\left(\frac{1}{n}-\frac{1}{m}\right)+C_{3} \sum_{s-\frac{1}{n}<t_{k}<s-\frac{1}{m}} c_{k}\right) \\
\int_{0}^{b} 1_{D_{m}}(s) \mathcal{M}\left(s, \mathbb{E}\left\|y_{n}\left(s-\frac{1}{m}\right)-y_{n}\left(s-\frac{1}{n}\right)\right\|^{2}\right. \\
\left.\quad+\mathbb{E}\left\|y_{n}\left(s-\rho(s)-\frac{1}{m}\right)-y_{n}\left(s-\rho(s)-\frac{1}{n}\right)\right\|^{2}\right) d s \\
\leqslant \mathcal{M}\left(s, 8 \mathbb{E}\|\varrho\|^{2}+8 C\right)\left(\frac{1}{n}+\frac{1}{m}\right) \\
\quad+b \mathcal{M}\left(s, 2 C_{1}\left\|S_{\beta}\left(\frac{1}{n}-\frac{1}{m}\right)-\mathbb{I}\right\|^{2}+2 C_{2}\left(\frac{1}{n}-\frac{1}{m}\right)+2 C_{3} \sum_{s-\frac{1}{n}<t_{k}<s-\frac{1}{m}} c_{k}\right)
\end{aligned}
$$

and:

$$
\begin{aligned}
& \mathbb{E}\left\|y_{n}\left(t_{k}-\frac{1}{m}\right)-y_{n}\left(t_{k}-\frac{1}{n}\right)\right\|^{2} \\
& \quad \leqslant C_{1}\left\|S_{\beta}\left(\frac{1}{n}-\frac{1}{m}\right)-\mathbb{I}\right\|^{2}+C_{2}\left(\frac{1}{n}-\frac{1}{m}\right)+C_{3} \sum_{s-\frac{1}{n}<t_{k}<s-\frac{1}{m}} c_{k} .
\end{aligned}
$$


Let

$$
Y(t)=\limsup _{m, n \longrightarrow \infty} 2 \mathbb{E}\left(\sup _{0 \leqslant s \leqslant t}\left\|y_{m}(s)-y_{n}(s)\right\|^{2}\right) .
$$

Since $\mu\left(D_{n}-D_{m}\right) \longrightarrow 0$ and $\mathcal{M}(s,)=$.0 as $n, m \longrightarrow \infty$, and employing (25)-(27), Equations (24) and (28) beside Fatou's lemma yield:

$$
Y(t) \leqslant 2 C_{4} \int_{0}^{t} \mathcal{M}(s, Y(s)) d s .
$$

Lastly, through Equation (29) and condition (A3), the following is obtained:

$$
Y(t)=\limsup _{m, n \longrightarrow \infty} 2 \mathbb{E}\left(\sup _{0 \leqslant s \leqslant t}\left\|y_{m}(s)-y_{n}(s)\right\|^{2}\right)=0,
$$

which yields:

$$
\limsup _{m, n \longrightarrow \infty} \mathbb{E}\left(\sup _{0 \leqslant s \leqslant t}\left\|y_{m}(s)-y_{n}(s)\right\|^{2}\right)=0,
$$

which shows that $\left\{y_{n}(t)\right\}_{n \geqslant 2 / \tau}$ is Cauchy sequence on $\mathcal{M}_{2}([-\tau, b] ; \mathcal{X})$. The Borel-Cantelli lemma shows that, as $n \longrightarrow \infty, y_{n}(t) \longrightarrow y(t)$ holds uniformly for every $t \in[0, b]$. Consequently, by the limit on both sides of (4), $y(t)$ defines the mild solution of (1) with property:

$$
\mathbb{E}\left(\sup _{0 \leqslant s \leqslant t}\|y(s)\|^{2}\right)=0, \quad 0 \leqslant t \leqslant b .
$$

Lastly, the proof of existence is complete. The uniqueness proof is presented in the Appendix A. Hence, the Theorem 1 proof is completed.

If $g(t, . ..) \equiv g\left(t, y_{t}\right), h(t, . ., \eta) \equiv h\left(t, y_{t}, \eta\right)$ and $f(t,.) \equiv f\left(t, y_{t}\right)$, Equation (1) reduces to the following equation:

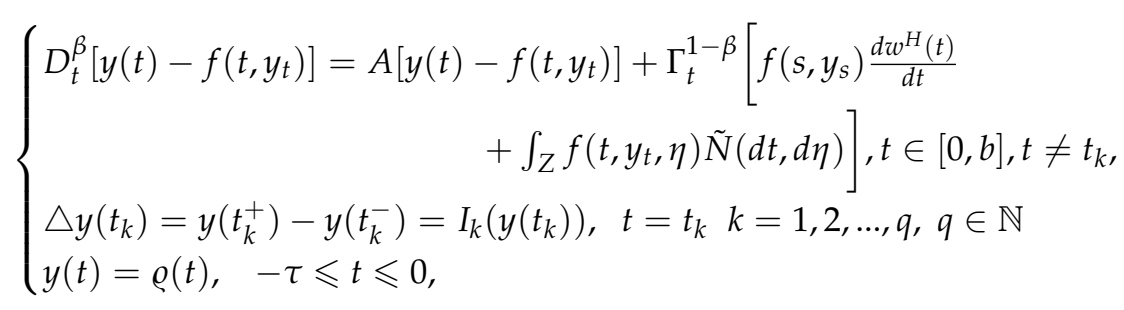

Corollary 1. Assume assumptions (A1)-(A5) are fulfilled. Suppose $\varrho$ is independent of the Poisson counting measure $\tilde{N}(d t, d \eta)$ and $f B m w^{H}(t)$. Then, provided $7 C_{f}+7 q M^{2} \sum_{k=1}^{q} c_{k}<1$, Equation (30) has a unique mild solution $y(t)$ on $\mathcal{M}_{2}([-\tau, b], \mathcal{X})$.

Remark 2. If $I_{k}(.) \equiv 0(k=1,2, \ldots, q)$ in Equation (30), Corollary 1 consists with Theorem 3.3 in Ramkumar et al. [21], where the authors applied the successive approximation to utilize existence and uniqueness problem under non-Lipschitz conditions. However, here, the results were utilized by using Carathéodory approximation under Carathéodory conditions with reference to [21] non-Lipschitz conditions as special case. So, Corollary 1 generalizes some of the results in [21]. 


\section{Application}

Consider the following IFNSPDEs driven by fBm and Poisson jumps:

$$
\left\{\begin{aligned}
& D_{t}^{\beta}[z(t, y)\left.-\int_{-\tau}^{0} \alpha_{1}(r) \sin z(t+r, y) d r\right]=\frac{\partial^{2}}{\partial y^{2}}\left[z(t, y)-\int_{-\tau}^{0} \alpha_{1}(r) \sin z(t+r, y) d r\right] \\
&\left.+\frac{1}{\Gamma(1-\beta}\right) \int_{0}^{t}(t-r)^{-\beta}\left[\int_{0}^{t} \int_{-\tau}^{t} e^{4(\tau-t)} z(r, y) d r d w^{H}(r)\right. \\
&\left.+\int_{Z} \eta\left(\int_{-\tau}^{t} \alpha_{2}(r-t) z(r, y) d r\right) \tilde{N}(d t, d \eta)\right] \\
& \triangle z\left(t_{k}, y\right):=z\left(t_{k}^{+}, y\right)-z\left(t_{k}^{-}, y\right)=\frac{\alpha_{3}}{2^{k}} z\left(t_{k}, y\right), \quad k \in \mathbb{N} \\
& y \in \mathcal{D}=(0, \pi], \quad t \in[0, b] \\
& z(t, 0)=z(t, \pi)=0, \quad t \in[0, T] \\
& z(t, y)=\varphi(t, y), \quad-\tau \leqslant t \leqslant 0, \quad y \in \mathbb{D}
\end{aligned}\right.
$$

with $D_{t}^{\beta}$, which is a fractional Caputo derivative of order $0<\beta<1$, and $w^{H}, \frac{1}{2}<H<1$ is $\mathrm{fBm}$. Let $\mathcal{X}=\mathcal{L}_{2}([0, \pi])$, and define the operator $A: \mathbb{D}(A) \subset \mathcal{X} \longrightarrow \mathcal{X}$ by $A z=\xi$ with domain $\mathbb{D}(A)=\{z \in \mathcal{X} ; z, z$ are absolutely continuous, $z \in \mathcal{X}, z(0)=z(\pi)=0\}$. Then, $A_{z}=\sum_{n=1}^{\infty} n^{2}\left(z, z_{n}\right) z_{n}, z \in \mathbb{D}(A)$, where $z_{n}(y)=\sqrt{\frac{2}{\pi}} \sin (n y), n \in \mathbb{N}$, which is a set of orthogonal eigenvectors of $A$. Define the $\mathrm{fBm}$ in $\mathcal{Y}$ by:

$$
w^{H}(t)=\sum_{n=1}^{\infty} \sqrt{\lambda_{n}} w_{n}^{H}(t) e_{n}
$$

where $\left\{w_{n}^{H}(t), n \in \mathbb{N}\right\}$ are standard mutually independent $\mathrm{fBms}$. Thanks to the subordination principle of solution operator, $A$ is the infinitesimal generator of a solution operator $\left\{S_{\beta}(t), t \geqslant 0\right\}$. Since $S_{\beta}(t)$ is strongly continuous on $[0, \infty)$ by a uniformly bounded theorem, there exists a constant $M>0$ such that $\left\|S_{\beta}(t)\right\|^{2} \leqslant M^{2}$, for $t \in[0, b]$. Define the nonlinear functions $f:[0, b] \times \wp \longrightarrow \mathcal{X}, g:[0, b] \times \wp \times \wp \longrightarrow \mathcal{L}_{2}^{0}(\mathcal{Y}, \mathcal{X})$ and $h:[0, b] \times \wp \times \wp \times Z \longrightarrow \mathcal{X}$ by:

$$
\begin{gathered}
f(t, \varrho)(y)=\int_{-\tau}^{0} \alpha_{1}(\zeta) \sin (\varrho(\zeta)(y)) d \zeta, \quad \zeta \in[-\tau, 0], y \in \mathbb{D}, \\
g(t, \psi, \varrho)(y)=\int_{-\tau}^{0} e^{-4 \zeta} \varrho(\zeta)(y) d \zeta, \\
h(t, \psi, \varrho)(y)=\int_{-\tau}^{0} \alpha_{2}(\zeta) \varrho(\zeta)(y) d \zeta, \\
I_{k}\left(y\left(t_{k}\right)\right)=\frac{\alpha_{3}}{2^{k}} z\left(t_{k}, y\right), \quad k \in \mathbb{N}
\end{gathered}
$$

and assuming that $\int_{Z} \eta^{2} \tilde{\lambda}(d \eta)<\infty$. Finally, system (31) takes the abstract form of model (1) and assumes that assumptions (A1)-(A5) are satisfied. Then, Theorem 1 guarantee that model (31) has a mild solution, which is unique.

\section{Conclusions}

Through this study, the existence of the unique mild solution for a class of IFNSDEs with variable delay driven by $\mathrm{fBm}$ and Poisson jumps was investigated in Hilbert space. Our model is more general than $[21,33]$ models as it takes the variable delays described by the term $\rho(t)$ and possible jumps shown as impulses into consideration. First, a new class of sufficient conditions for the existence of mild solutions of the aforementioned class of equations was established, which is more general than the non-Lipschitz condition used in [21] and contains it as special case. The existence results were formulated and proved by using a solution operator, fractional calculus, the Carathéodory approximation approach, and stochastic analysis techniques. An application was provided to validate the obtained 
theoretical results. Our results improve and enhance some results in [21]. Our future work is to consider the problem of an averaging principle for IFNSDEs with variable delay under $\mathrm{fBm}$ and Poisson-jump perturbations.

Author Contributions: Formal analysis, M.A. and R.A.R.B.; Methodology, M.A. and W.A.; Writingoriginal draft, M.A. and M.E.; Writing—review and editing, M.A. and A.D.K. Authors equally contributed to the preparation of this manuscript. All authors have read and agreed to the published version of the manuscript.

Funding: This research was funded by the Deanship of Scientific Research of the University of King Abdulaziz (No. FP-044-43).

Institutional Review Board Statement: Not applicable.

Informed Consent Statement: Not applicable.

Data Availability Statement: The current article did not produce any datasets.

Acknowledgments: We are very grateful to the anonymous referees for their suggestions and valuable advice. The Deanship of Scientific Research of King Abdulaziz University (No. FP-044-43) supported the research.

Conflicts of Interest: No competing interest are declared by the authors.

\section{Appendix A}

Proof of the Uniqueness: Let $x(t), y(t)$ be two solutions of Equation (1). Then, the uniqueness is obvious on the interval $[-\tau, 0]$, and for $t \in[0, b]$, by similar analysis of Equation (18), elementary inequality, Lemma 1, Burkholder's inequality, and conditions (A1), (A3), and (A5), it is easy to obtain:

$$
\begin{aligned}
& \mathbb{E}\left(\sup _{0 \leqslant s \leqslant t}\|x(s)-y(s)\|^{2}\right) \\
& \leqslant \frac{12 M^{2} H t^{2 H-1}}{1-2 c_{f}} \int_{0}^{t} \mathbb{E}\|g(s, x(s), x(s-\rho(s)))-g(s, y(s), y(s-\rho(s)))\|^{2} d s \\
& \quad+\frac{6 M^{2} C_{b}}{1-2 c_{f}}\left[\mathbb{E} \int_{0}^{t} \int_{Z}\|h(s, x(s), x(s-\rho(s)), \eta)-h(s, y(s), y(s-\rho(s)), \eta)\|^{2} \tilde{\lambda}(d \eta) d s\right. \\
& \left.+\mathbb{E}\left(\int_{0}^{t} \int_{Z}\|h(s, x(s), x(s-\rho(s)), \eta)-h(s, y(s), y(s-\rho(s)), \eta)\|^{4} \tilde{\lambda}(d \eta) d s\right)^{\frac{1}{2}}\right] \\
& +\frac{6 q M^{2}}{1-2 c_{f}} \sum_{k=1}^{q} \mathbb{E}\left\|I_{k}\left(x\left(t_{k}\right)\right)-I_{k}\left(y\left(t_{k}\right)\right)\right\|^{2} \\
& \leqslant \frac{6 M^{2}\left(C_{b}+2 H t^{2 H-1}\right)}{1-2 C_{f}-6 q M^{2} \sum_{k=1}^{q} c_{k}} \int_{0}^{t} \mathcal{M}\left(s, 2 \mathbb{E} \sup _{0 \leqslant u \leqslant s}\|x(u)-y(u)\|^{2}\right) d s .
\end{aligned}
$$

Then:

$2 \mathbb{E}\left(\sup _{0 \leqslant s \leqslant t}\|x(s)-y(s)\|^{2}\right) \leqslant \frac{24 M^{2}\left(C_{b}+H t^{2 H-1}\right)}{1-2 C_{f}-6 q M^{2} \sum_{k=1}^{q} c_{k}} \int_{0}^{t} \mathcal{M}\left(s, 2 \mathbb{E} \sup _{0 \leqslant u \leqslant s}\|x(u)-y(u)\|^{2}\right) d s$,

which, with the aid of condition (A3.c), gives:

$$
\mathbb{E}\left(\sup _{0 \leqslant s \leqslant t}\|x(s)-y(s)\|^{2}\right)=0, \quad 0 \leqslant t \leqslant b
$$

Therefore, $x(t)=y(t)$ for all $0 \leqslant t \leqslant b$. Hence, the uniqueness is proved. 


\section{References}

1. Mostaghim, Z.S.; Moghaddam, B.P.; Haghgozar, H.S. Computational technique for simulating variable-order fractional Heston model with application in US stock market. Math. Sci. 2018, 12, 277-283. [CrossRef]

2. Agarwal, P.; Wang, G.; Al-Dhaifallah, M. Fractional calculus operators and their applications to thermal systems. Adv. Mech. Eng. 2018, 10. [CrossRef]

3. Samko, S.G.; Kilbas, A.A.; Marichev, O.I. Fractional Integrals and Derivatives: Theory and Applications; Gordon and Breach: London, UK, 1993.

4. Miller, K.S.; Ross, B. An Introduction to Fractional Calculus and Differential Equations; John Wiley: New York, NY, USA, 1993.

5. Kilbas, A.A.; Srivastava, H.M.; Trujillo, J.J. Theory and Applications of Fractional Differential Equations; Elsevier Science: Amsterdam, The Netherlands, 2006.

6. Tien, D.N. Fractional stochastic differential equations with applications to finance. J. Math. Anal. Appl. 2013, 397, 334-348. [CrossRef]

7. Yu., Z.-G.; Anh, V.; Wang, Y.; Mao, D.; Wanliss, J. Modeling and simulation of the horizontal component of the geomagnetic field by fractional stochastic differential equations in conjunction with empirical mode decomposition. J. Geophys. Res. Space Phys. 2010, 115, A10219. [CrossRef]

8. Abdel-Rehim, E. From the Ehrenfest model to time-fractional stochastic processes. J. Comput. Appl. Math. 2009, 233, 197-207. [CrossRef]

9. Zou, G.; Wang, B. On the study of stochastic fractional-order differential equation systems. arXiv 2016, arXiv:1611.07618.

10. Moghaddam, B.P.; Zhang, L.; Lopes, A.M.; Tenreiro Machado, J.A.; Mostaghim, Z.S. Sufficient conditions for existence and uniqueness of fractional stochastic delay differential equations. Stochastics 2020, 92, 379-396. [CrossRef]

11. Li, K. Stochastic delay fractional evolution equations driven by fractional Brownian motion. Math. Meth. Appl. Sci. 2015, 38, 1582-1591. [CrossRef]

12. Xu, L.; Li, Z. Stochastic fractional evolution equations with fractional brownian motion and infinite delay. Appl. Math. Comput. 2018, 336, 36-46. [CrossRef]

13. Abouagwa, M.; Liu, J.; Li, J. Carathéodory approximations and stability of solutions to non-Lipschitz stochastic fractional differential equations of Itô-Doob type. Appl. Math. Comput. 2018, 329, 143-153. [CrossRef]

14. Abouagwa, M.; Li, J. Approximation properties for solutions to Itô-Doob stochastic fractional differential equations with non-Lipschitz coefficients. Stoch. Dyn. 2019, 19, 1950029. [CrossRef]

15. Rihan, F.A.; Rajivganthi, C.; Muthukumar, P. Fractional stochastic differntial equations with Hilfer fractional derivative: Poisson jumps and optimal control. Discret. Dyn. Nat. Soc. 2017, 2017, 11. [CrossRef]

16. Balasubramaniam, P.; Ratnavelu, K.; Saravanakumar, S. Study a class of Hilfer fractional stochastic integrodifferential equations with Poisson jumps. Stoch. Anal. Appl. 2018, 36, 1021-1036. [CrossRef]

17. Yang, M.; Gu, H. Riemann-Liouville fractional stochastic evolution equations driven by both Wiener process and fractional Brownian motion. J. Inequl. Appl. 2021, 2021, 8. [CrossRef]

18. Luo, J.; Taniguchi, T. The existence and uniqueness for non-Lipschitz stochastic neutral delay evolution equations driven by Poisson jumps. Stoch. Dyn. 2009, 9, 135-152. [CrossRef]

19. Lakhel, E.; McKibben, M.A. Existence of solutions for fractional neutral functional differential equations driven by fBm with infinite delay. Stochastics 2018, 90, 313-329. [CrossRef]

20. Dhanalakshmi, K.; Balasubramaniam, P. Stability result of higher-order fractional neutral stochastic differential system with infinite delay driven by Poisson jumps and Rosenblatt process. Stoch. Anal. Appl. 2020, 38, 352-372. [CrossRef]

21. Ramkumar, K.; Ravikumar, K.; Varshini, S. Fractional neutral stochastic differential equations with Caputo fractional derivative: Fractional Brownian motion, Poisson jumps, and optimal control. Stoch. Anal. Appl. 2021, 39, 157-176. [CrossRef]

22. Alnafisah, Y.; Ahmed, H.M. Neutral delay Hilfer fractional integrodifferential equations driven with fractional Brownian motion. Evol. Equ. Control Theory 2021. [CrossRef]

23. Girel, S.; Crauste, F. Existence and stability of periodic solutions of an impulsive differential equation and application to CD8 T-cell differentiation. J. Math. Biol. 2018, 76, 1765-1795. [CrossRef]

24. Catllá, A.J.; Schaeffer, D.G.; Witelski, T.P.; Monson, E.E.; Lin, A.L. On spiking models for synaptic activity and impulsive differential equations. SIAM Rev. 2008, 50, 553-569. [CrossRef]

25. Li, X.D.; Bohner, M.; Wang, C.K. Impulsive differential equations: Periodic solutions and applications. Automatica 2015, 52, 173-178. [CrossRef]

26. Dhayal, R.; Malik, M. Existence and controllability of impulsive fractional stochastic differential equations driven by Rosenblatt process with Poisson jumps. J. Eng. Math. 2021, 130, 11. [CrossRef]

27. Balasubramanian, P.; Kumaresan, N.; Ratnavelu, K.; Tamilalgan, P. Local and global existence of mild solution for impulsive fractional stochastic differential equations. Bull. Malays. Math. Sci. Soc. 2015, 38, 867-884. [CrossRef]

28. Saravanakumar, S.; Balasubramaniam, P. Non-instantaneous impulsive Hilfer fractional stochastic differential equations driven by fractional Brownian motion. Stoch. Anal. Appl. 2021, 39, 549-566. [CrossRef]

29. Abouagwa, M.; Cheng, F.; Li, J. Impulsive stochastic fractional differential equations driven by fractional Brownian motion. Adv. Differ. Equ. 2020, 2020, 57. [CrossRef] 
30. Anguraj, A.; Ravikumar, K. Existence and stability results for impulsive stochastic functional integrodifferential equations with Poisson jumps. J. Appl. Nonlinear Dyn. 2019, 8, 407-417. [CrossRef]

31. Dhanalakshmi, K.; Balasubramaniam, P. Exponential stability of impulsive fractional neutral stochastic differential equations. J. Math. Phys. 2021, 62, 092703. [CrossRef]

32. Muthukumar, P.; Thiagu, K. Existence of solution and approximate controllability of fractional neutral impulsive stochastic differential equation of order $1<q \leqslant 2$ with infinite delay and Poisson jumps. Differ. Equ. Dyn. Syst. 2018, 26, 15-36.

33. Taniguchi, T. Successive approximations to solutions of stochastic differential equations. J. Differ. Equ. 1992, 96, 152-169. [CrossRef]

34. Mandelbrot, B.B.; Van Ness, J.W. Fractional Brownian motions, fractional noises and applications. SIAM Rev. 1968, 10, 422-437. [CrossRef]

35. Alos, E.; Mazet, O.; Nualart, D. Stochastic Calulus with respect to Gaussian processes. Ann. Probab. 1999, $29,766-801$.

36. Mishura, Y. Stochastic Calulus for Fractional Brownian Motion and Retarted Topics. In Lecture Notes in Mathematics; Springer Science, Business Media: Berlin/Heidelberg, Germany, 2008; Volume 1929.

37. Nualart, D. The Malliavin Calculus and Related Topics, 2nd ed.; Springer: Berlin, Germany, 2006.

38. Khalaf, A.D.; Zeb, A.; Saeed, T.; Abouagwa, M.; Djilali, S.; Alshehri, H. A special study of the mixed weighted fractional Brownian motion. Fractal Fract. 2021, 5, 192. [CrossRef]

39. Podlubny, I. Fractional Differential Equations. Mathematics in Sciences and Engineering; Academic Press: London, UK, 1999.

40. Ren, Y.; Zhou, Q.; Chen, L. Existence, uniqueness and stability of mild solutions for time-dependent stochastic evolution equations with Poisson jumps and infinite delay. J. Optim. Theory Appl. 2011, 149, 315-331. [CrossRef] 